\title{
GSK is to stop paying doctors to talk about its drugs and attend conferences
}

This News story (BMJ 2013;347:f7579, doi:10.1136/bmj.f7579) was originally published on 17 December 2013, however, owing to a production error the article had to be republished and 